Supporting Information

\title{
Single Cell-Like Systems Reveal Active Unidirectional and Light-Controlled Transport by Nanomachineries
}

Tim Diederichs ${ }^{1}$, Robert Tampé ${ }^{1 *}$

${ }^{1}$ Institute of Biochemistry, Biocenter, Goethe University Frankfurt, Max-von-Laue-Str. 9, 60438 Frankfurt a.M., Germany 


\section{Content}

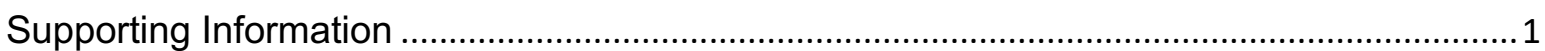

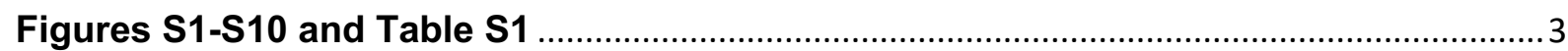




\section{Figures S1-S10 and Table S1}
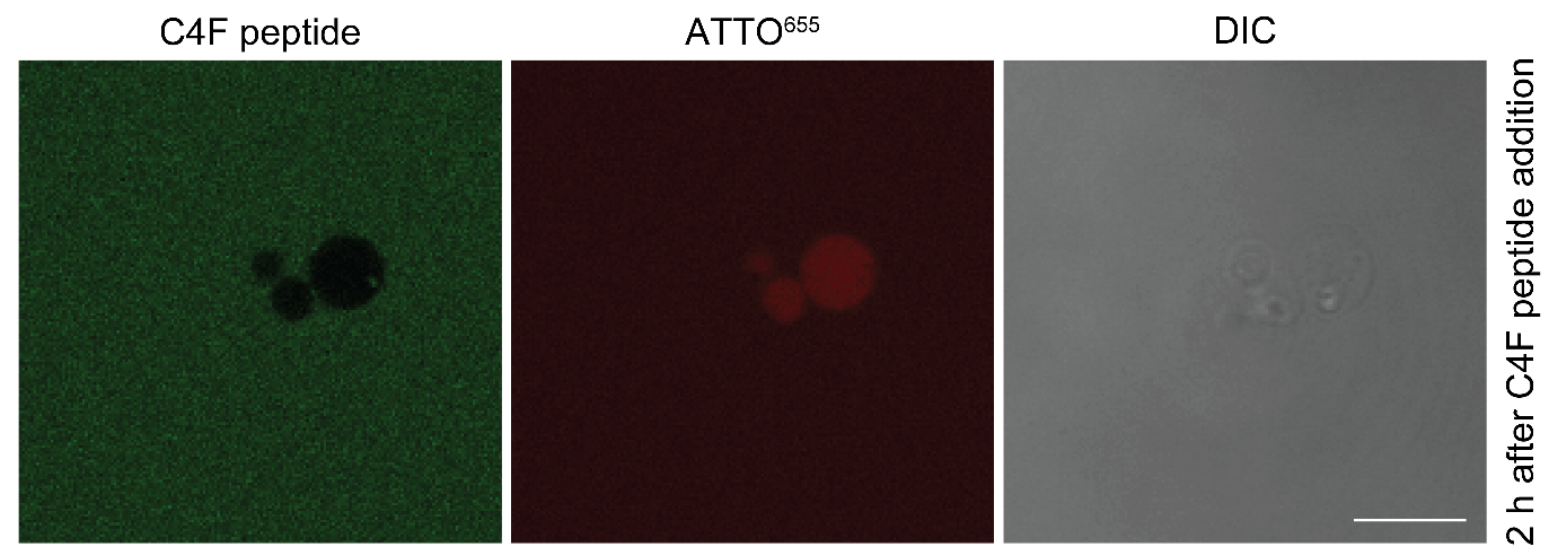

Figure S1: Membrane integrity of giant liposomes containing coreTAPL ${ }^{\text {menus. }}$. The fluorophore ATTO $^{655}$ was encapsulated in giant liposomes containing coreTAPL ${ }^{m V e n u s}$. The fluorescently labeled peptide $(\mathrm{C} 4 \mathrm{~F}, 3 \mu \mathrm{M})$ was added and the GUVs were imaged after $2 \mathrm{~h}$ incubation. Fluorescence intensities were enhanced for better visualization. Scale bar, $10 \mu \mathrm{m}$. 

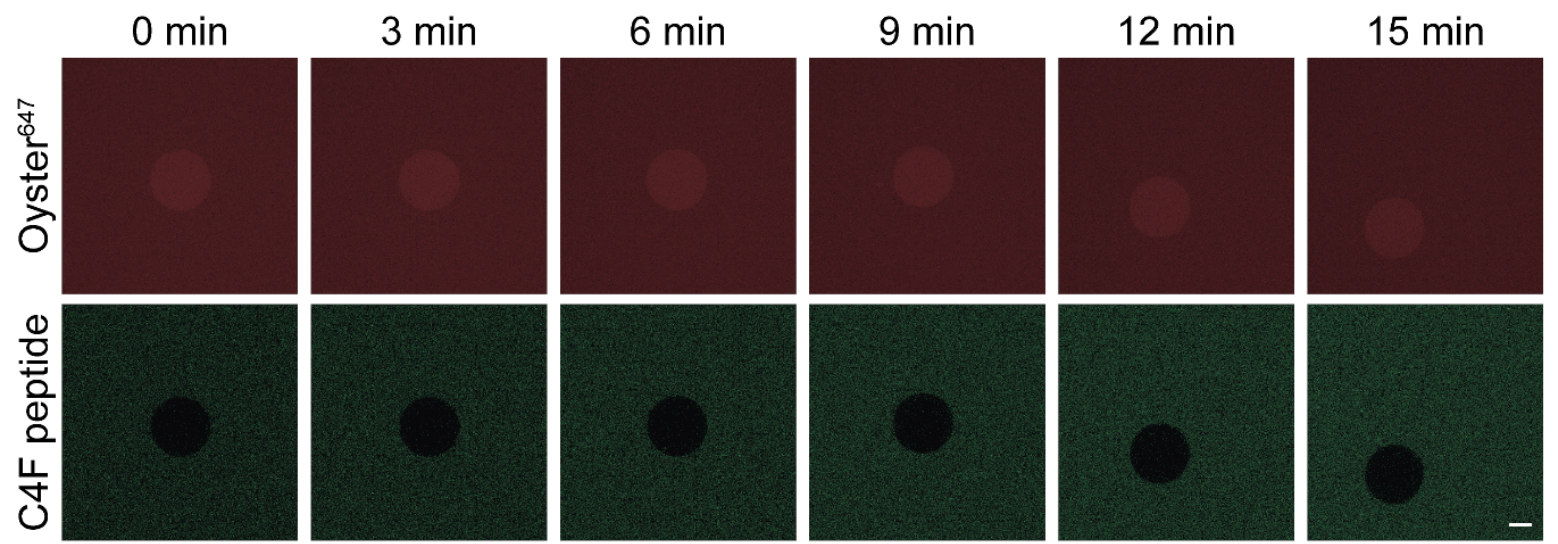

Figure S2: Membrane integrity of a giant proteoliposome before transport initiation by ATP. ProteoGUVs with a protein to lipid ratio of $1: 15 \mathrm{w} / \mathrm{w}$ were prepared and Oyster ${ }^{647}$ was encapsulated in the interior. The fluorescently labeled peptide $(\mathrm{C} 4 \mathrm{~F})$ and phosphocreatine were added, followed by imaging via confocal microscopy. Fluorescence intensities were enhanced for better visualization. Scale bar, $10 \mu \mathrm{m}$. 


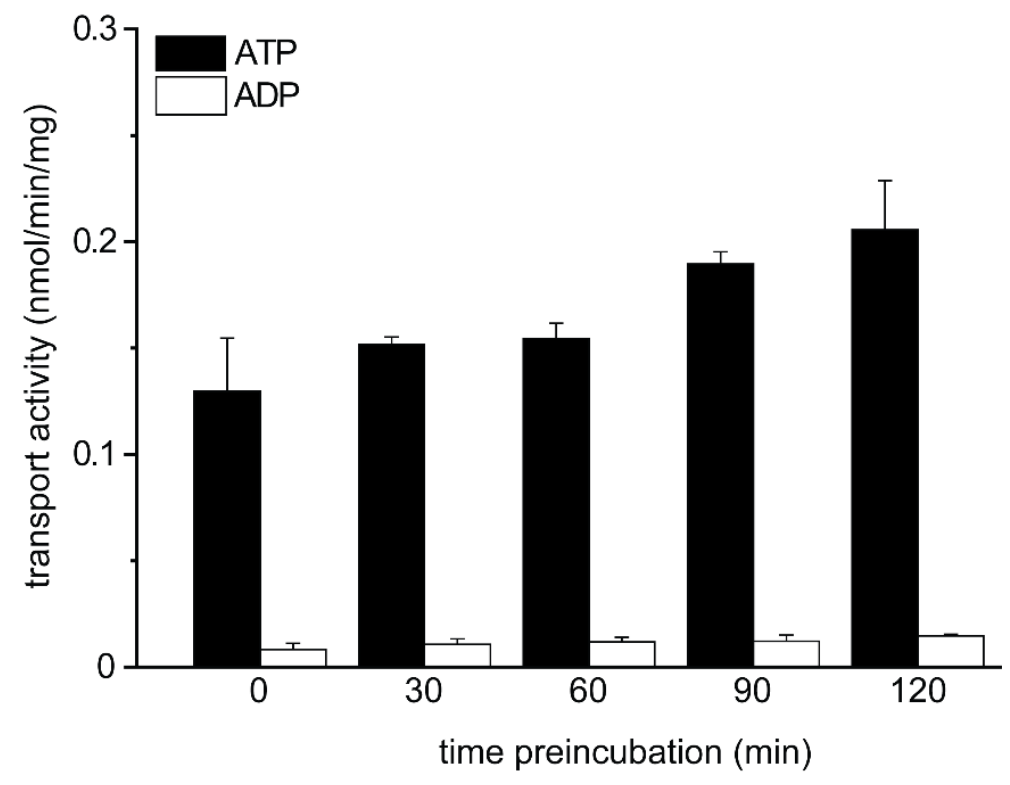

Figure S3: Long-term activity of TrmAB. The long-term activity of TmrAB reconstituted in liposomes (protein to lipid ratio 1:15w/w) was assessed by preincubation of proteoliposomes at $40{ }^{\circ} \mathrm{C}$, followed by a transport assay for $15 \mathrm{~min}$ in the presence of ATP and ADP ( $3 \mathrm{mM}$ each) and $3 \mu \mathrm{M} \mathrm{C} 4 \mathrm{~F}$. The mean $\pm \mathrm{SD}(n=3)$ is shown. 
a

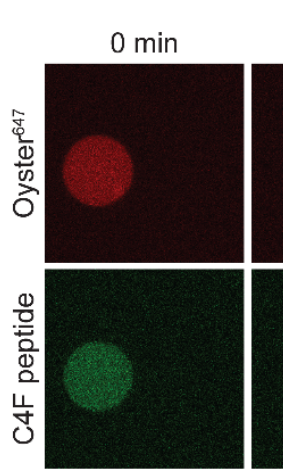

$3 \min$

$6 \min$

$9 \min$
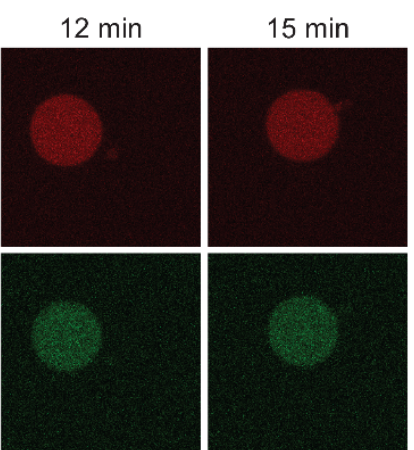

$18 \min$

b

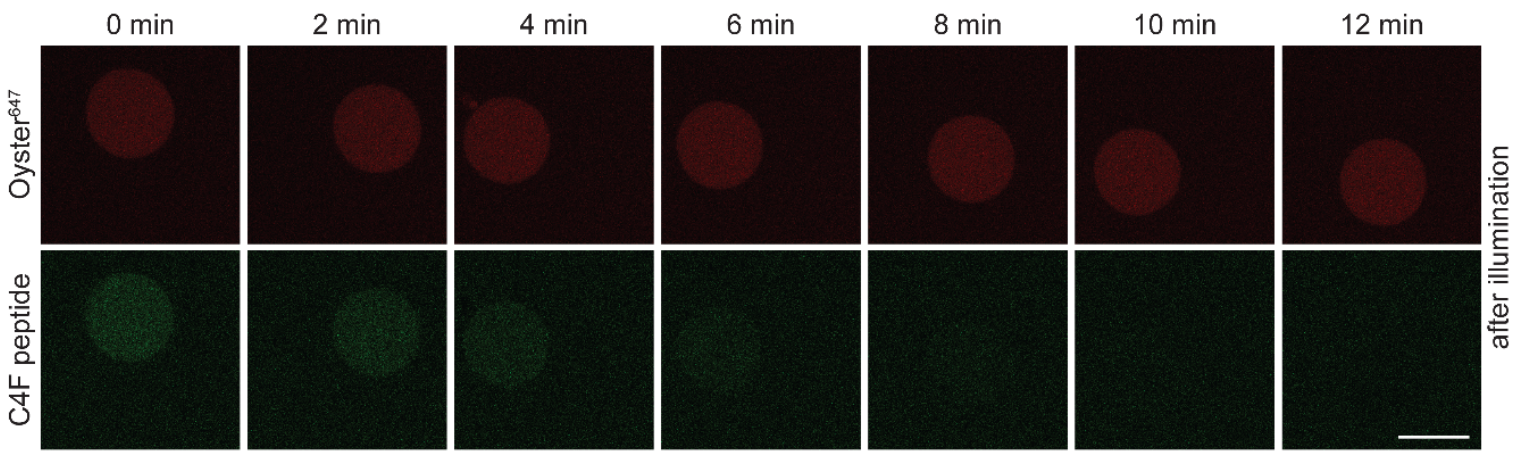

Figure S4: Direct comparison of a giant proteoliposome before and after photoactivation of caged-ATP. Oyster ${ }^{647}, \mathrm{C} 4 \mathrm{~F}, \mathrm{ARS}$, and photocleavable ATP were encapsulated in the GUV lumen. $\mathbf{a}$, Time-lapse images without photoactivation $(405 \mathrm{~nm})$. Scale bar, $20 \mu \mathrm{m}$. b. Time-lapse images after photoactivation of caged-ATP. Fluorescence intensities were enhanced for better visualization. Scale bar, $20 \mu \mathrm{m}$. 

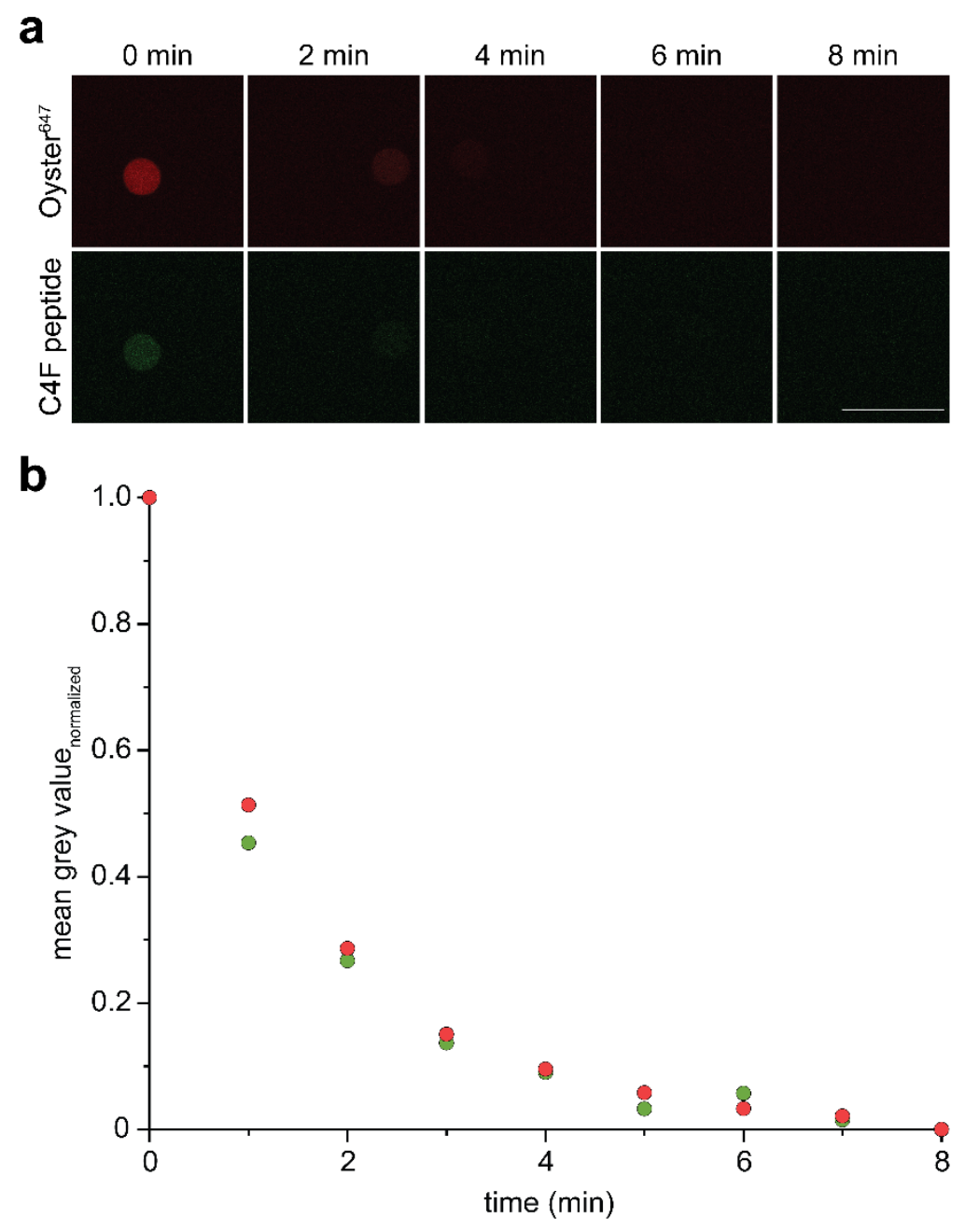

Figure S5: Example of rarely observed membrane leakiness events after in situ photoactivation. Oyster $^{647}$, C4F, ARS, and photocleavable ATP were encapsulated in the GUV lumen. a, Time-lapse images after photoactivation $(405 \mathrm{~nm})$ revealed membrane leakiness clearly distinguishable from protein-mediated efflux. Scale bar, $40 \mu \mathrm{m}$. b, Normalized mean grey analysis of time-lapse recordings as shown in (a). Monoexponential efflux of Oyster ${ }^{647}$ (red sphere) and C4F peptide (green sphere) reveal membrane leakiness, which is clearly distinguishable from TmrAB-mediated peptide export. In general, leakiness events are rarely observed. 


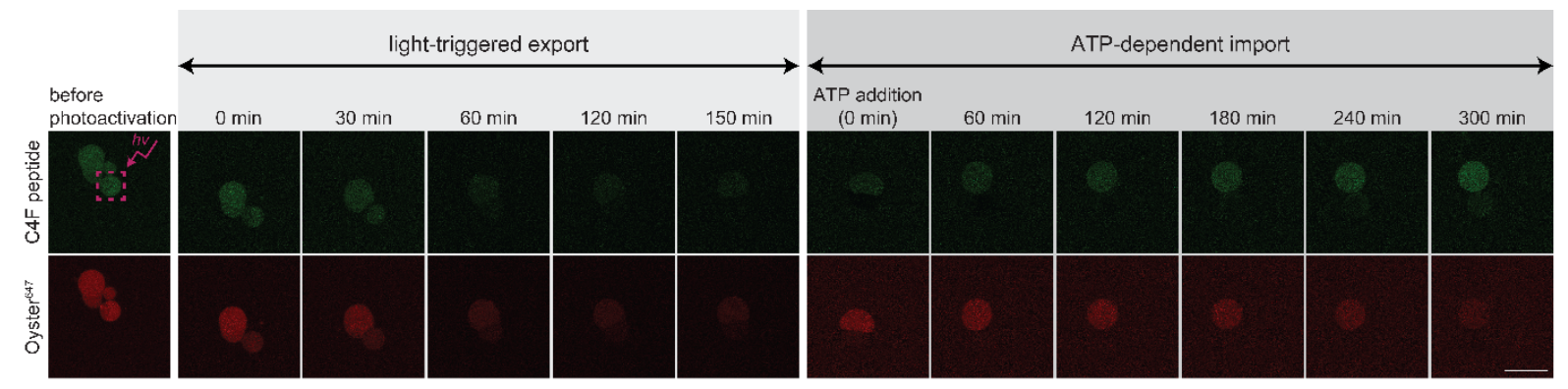

Figure S6: Export specificity and import of an individual in situ activated GUV. Oyster ${ }^{647}(10 \mu \mathrm{M})$, C4F $(20 \mu \mathrm{M})$, ARS, and photocleavable ATP $(2 \mathrm{mM})$ were encapsulated in the GUV lumen. The marked liposome (red dashed square) is illuminated by $405 \mathrm{~nm}$ followed by time-lapse recordings. After export, the focus was readjusted and the Oyster channel was enhanced for better visualization. Additionally, $2 \mathrm{mM}$ ATP and ARS were added from the outside. The diluted C4F concentration (remaining from the encapsulation) is transported in the inside of the marked vesicle over time. Scale bar, $20 \mu \mathrm{m}$. Other GUVs surrounding the photobleached GUV are moving in the z-direction or become leaky. 


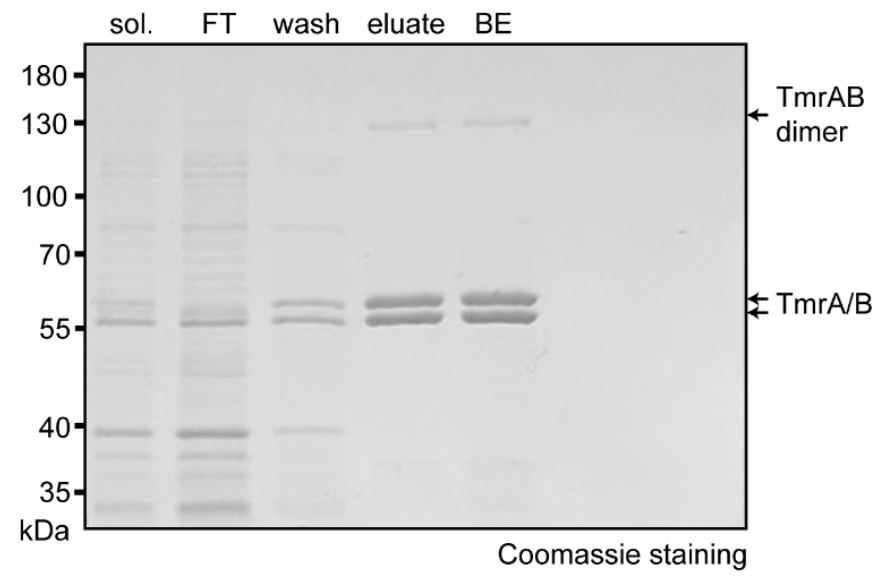

Figure S7: TmrAB purification. TmrAB expressed in E. coli cells were purified by immobilized metal affinity chromatography (IMAC) and a sample were pooled for each purification step (solubilization (sol.), flow through (TF), wash, eluate, and buffer exchange (BE)) and analyzed by SDS-PAGE and Coomassie staining. 
a

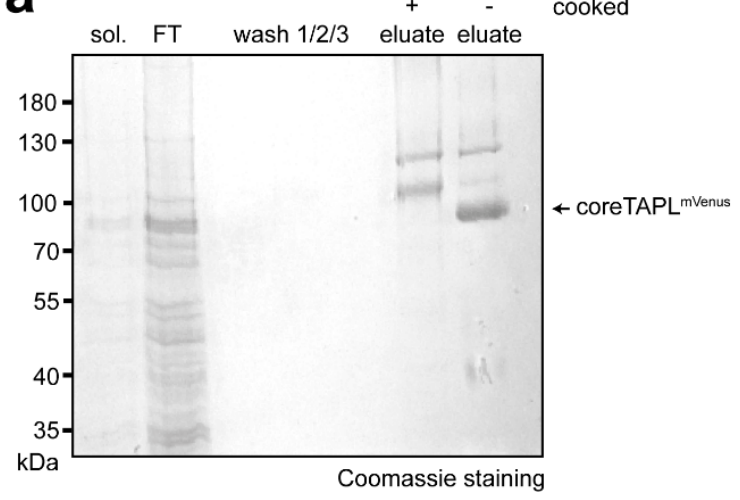

b

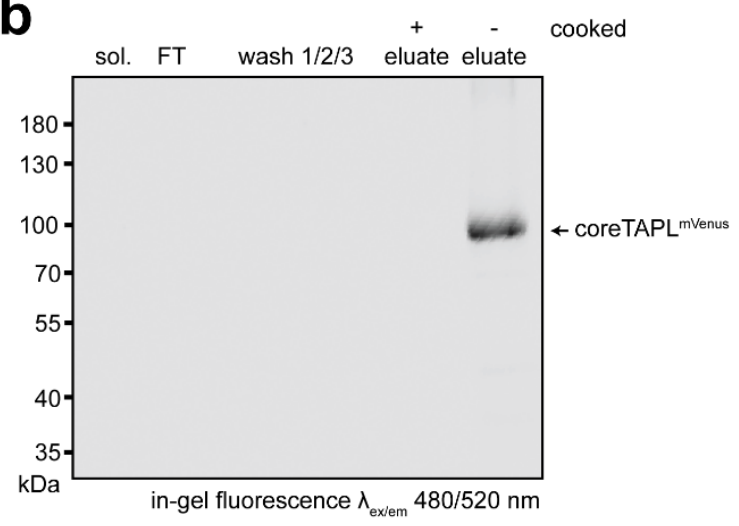

Figure S8: CoreTAPL ${ }^{\text {mvenus }}$ purification. Human coreTAPL ${ }^{\text {mVenus }}$ was expressed in Pichia pastoris and purified by IMAC. A sample for each purification step (solubilization (sol.), flow through (FT), wash1/2/3, eluate uncooked and cooked) was pooled and analyzed by SDS-PAGE. a, Coomassie staining. b, In-gel fluorescence of mVenus. 


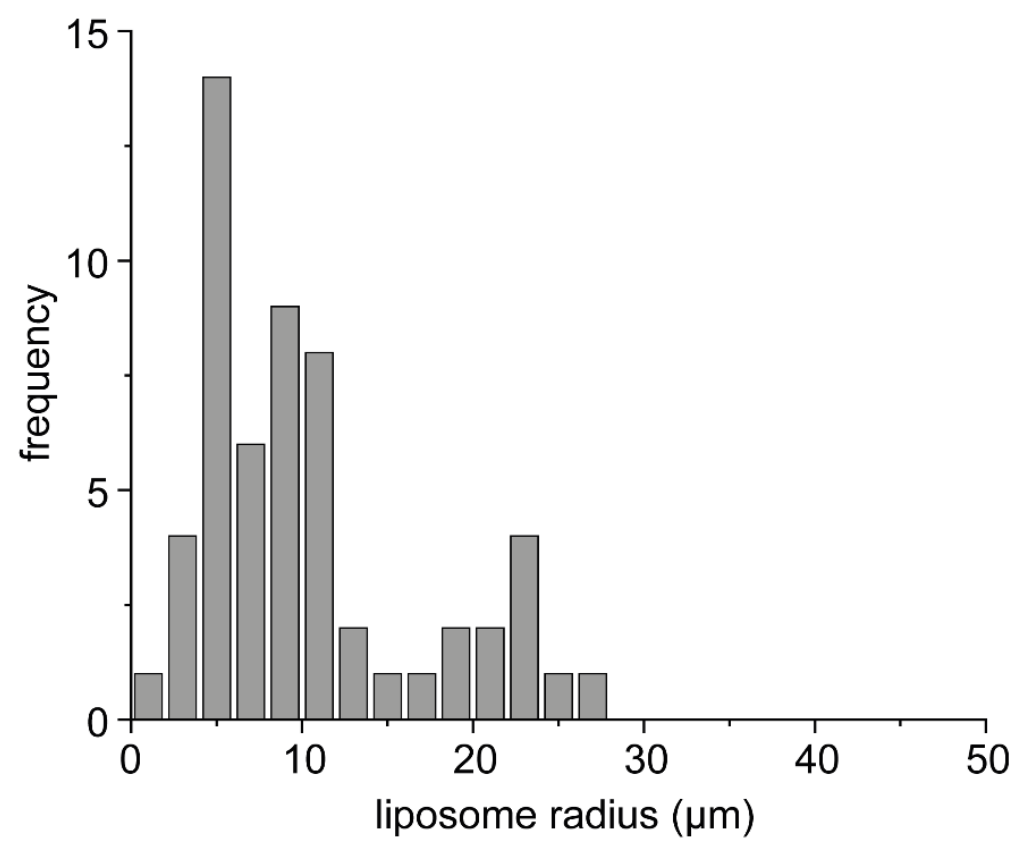

Figure S9: Size distribution of giant liposomes. The proteoGUV radii $(n=56)$ of the export and import experiments were determined and summarized in a histogram. A relatively broad distribution varying between $\sim 2 \mu \mathrm{m}$ and $\sim 28 \mu \mathrm{m}$, with two peaks were determined. 


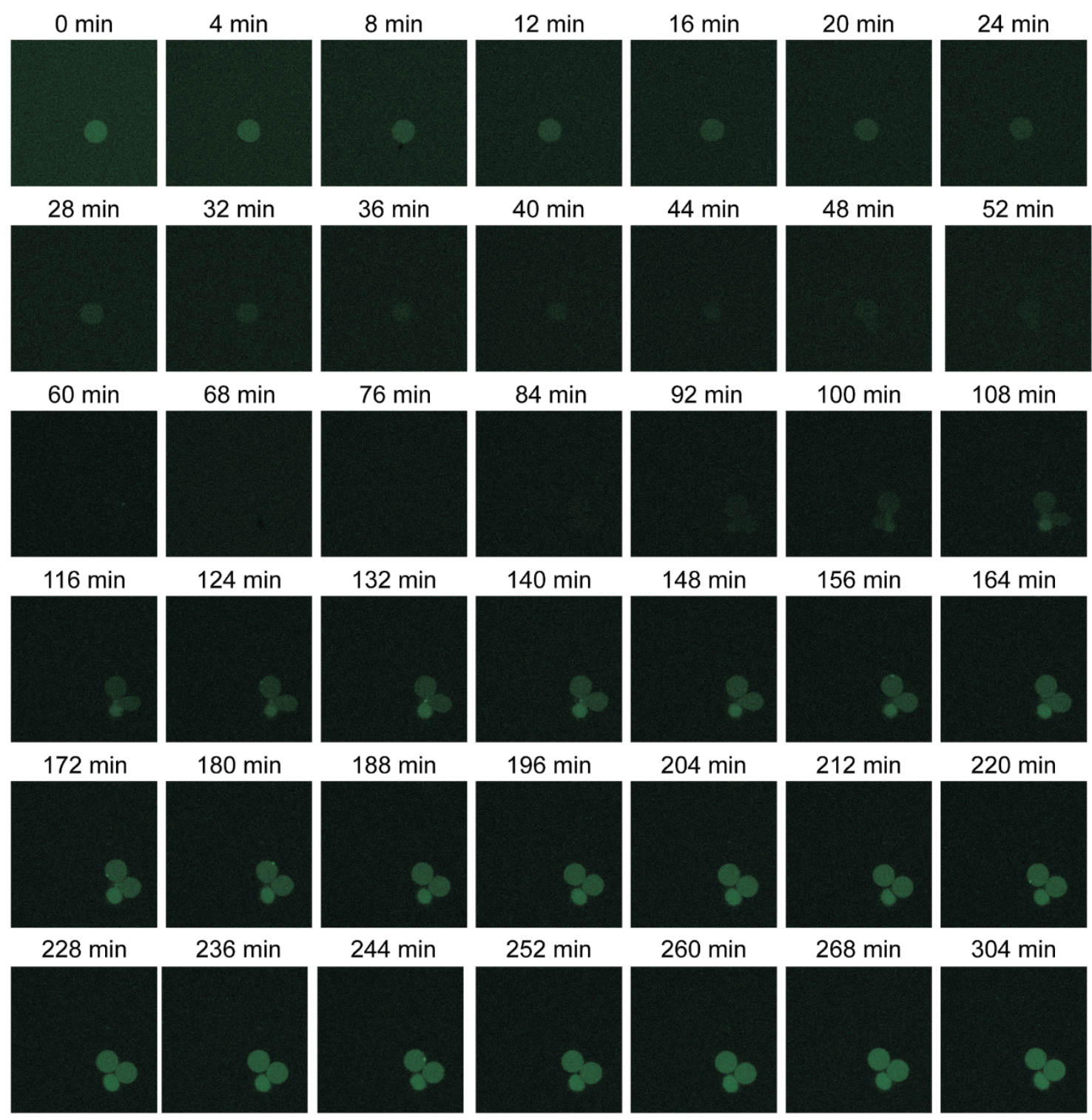

Figure S10: Export and import experiment (images of Figure 4e). Images of the C4F channel for the export/import experiment. 
Table S1: Summary of used substances for the export, import, and export / import assays. Three different conditions varying in the C4F concentration were applied as import experiments and the export/import experiment was performed with two C4F concentrations.

\begin{tabular}{|c|c|c|c|c|c|c|c|c|c|c|c|c|}
\hline & \multicolumn{6}{|c|}{ import } & \multirow{2}{*}{\multicolumn{2}{|c|}{ export }} & \multicolumn{4}{|c|}{ export / import } \\
\hline & \multicolumn{2}{|c|}{$1^{\text {st }}$} & \multicolumn{2}{|c|}{$2^{\text {nd }}$} & \multicolumn{2}{|c|}{$3^{\text {rd }}$} & & & & $1^{\text {st }}$ & & $2^{\text {nd }}$ \\
\hline & in & out & in & out & in & out & in & out & in & out & in & out \\
\hline glucose (mM) & - & 200 & - & 200 & - & 200 & - & 200 & - & 200 & - & 200 \\
\hline sucrose (mM) & 200 & - & 200 & - & 200 & - & 200 & - & 200 & - & 200 & - \\
\hline HEPES pH 7.4 (mM) & 20 & 20 & 20 & 20 & 20 & 20 & 20 & 20 & 20 & 20 & 20 & 20 \\
\hline $\mathrm{NaCl}(\mathrm{mM})$ & 150 & 150 & 150 & 150 & 150 & 150 & 150 & 150 & 150 & 150 & 150 & 150 \\
\hline $\mathrm{MgCl}_{2}(\mathrm{mM})$ & 1 & 1 & 1 & 1 & 1 & 1 & 1 & 1 & 1 & 1 & 1 & 1 \\
\hline Oyster647 ( $\mu \mathrm{M})$ & 10 & - & 10 & - & 10 & - & 10 & - & 10 & - & 10 & - \\
\hline $\begin{array}{c}\text { phosphocreatine } \\
\text { (mM) }\end{array}$ & - & 2 & - & 2 & - & 2 & 2 & 2 & 2 & 2 & 2 & 2 \\
\hline $\begin{array}{l}\text { phosphocreatine } \\
\text { kinase (g/L) }\end{array}$ & - & 0.3 & - & 0.3 & - & 0.3 & 0.1 & - & 0.1 & $0.3^{*}$ & 0.1 & $0.3^{*}$ \\
\hline $\mathrm{C} 4 \mathrm{~F}(\mu \mathrm{M})$ & - & 0.2 & - & 1 & - & 3 & $10 / 20$ & - & 10 & residual ${ }^{\#}$ & 20 & residual $^{\#}$ \\
\hline ATP (mM) & - & 2 & - & 2 & - & 2 & - & - & - & $1^{*}$ & - & $1^{*}$ \\
\hline caged-ATP (mM) & - & - & - & - & - & - & 2 & - & 2 & - & 2 & - \\
\hline
\end{tabular}

*Substances were added after the export experiment. \#The C4F concentration is residual due to dilution after encapsulation. 